\title{
DFTB-MD Simulation of Shocked Water Cluster
}

\author{
Hongtao Liu ${ }^{1, \text { a }}$, Ping Zhou ${ }^{1, \mathrm{~b}}$, Hongjian $\mathrm{Li}^{1}$, Anyang $\mathrm{Li}^{2}$ and Yusheng Dou ${ }^{1}$ \\ ${ }^{1}$ Department of Computer Science and Technology, Chongqing University of Posts and \\ Telecommunications, Chongqing, 400065, China
}

${ }^{2}$ Key Laboratory of Synthetic and Natural Functional Molecule Chemistry of the Ministry of Education, College of Chemistry and Materials Science, Northwest University, Xi'an, 710069, China

aliuht@cqupt.edu.cn, b1249248935@qq.com

Keywords: multiscale shock technique, water, DFTB, quantum molecular dynamics

\begin{abstract}
Relatively efficient and precise quantum molecular dynamics simulations were performed to gain fundamental insights into the mechanisms for the primary detonation process of water under shock wave loading using self-consistent charge density-functional tight binding (SCC-DFTB) calculations combined with the multiscale shock technique (MSST) as well as DFTB+ program conjunct with MSST. We observe that water achieves chemical equilibrium in less than 4ps for all shock conditions studied. What's more, we make comparison with the experimental results for the Hugoniot pressure and density final states. At last, our simulations show that decomposition occurs through the reversible $\mathrm{H}_{2} \mathrm{O} \leftrightarrow \mathrm{H}^{+}+\mathrm{OH}^{-}$, in agreement with experiment. Therefore, the molecular dynamics method of water under extreme conditions is effective.
\end{abstract}

\section{Introduction}

In recent years, the nature of water under extreme conditions has gradually been widely concerned. For example, subcritical water and supercritical water can be used as clean and efficient reaction medium or reactant. ${ }^{[1]}$ In spite of intense experimental research ${ }^{[2]}$, the coverage of the nature of water under high temperature and pressure is still rarely. Diamond anvil cell experiments have successfully studied the state of water under high pressure and low temperature, as well as the melting line of compressed ice under lower pressure and high temperature. However, the thermodynamic state, which can't be accessible in DAC experiments, can be obtained by shock compression. Previous report has showed that shock can induce melting and freezing of ices at relatively low pressures. What's more the pressures can conduct up to 790GPa with laser driven shock compression on liquid water. ${ }^{[3]}$ However, it has been showed that the temperatures deduced by equation of state models can overestimate that measured in the shock compression experiments by approximately $17 \% .{ }^{[4]}$ In addition, the chemical mechanisms for ionic transport in shock compressed water still have differences. Shock compression experiments on water have showed that charge transport comes up through diffusion of $\mathrm{H}^{+}$ions, created by a unimolecular dissociation mechanism, $\mathrm{H}_{2} \mathrm{O} \leftrightarrow \mathrm{H}^{+}+\mathrm{OH}^{-[5]}$. On the contrary, it has been predicted in not shocked simulations that proton is produced through a bimolecular dissociation mechanism, $2 \mathrm{H}_{2} \mathrm{O} \leftrightarrow \mathrm{OH}^{-}+\mathrm{H}_{3} \mathrm{O}^{+}$. ${ }^{[6]}$ Thus, for the reason of high cost of the experiments under extreme conditions, experiments can benefit from the molecular dynamics simulations.

\section{Computational Details}

Recently, it has been extremely difficult to obtain a clear theoretical mechanism of chemistry behind shock fronts because of the large system size required for shock simulation. [7] Empirical [8] and tight-binding [9] simulations have been successfully applied to several reactive systems of shock compression. In molecular dynamics simulations, it is commonly to adopt quantum theory to simulate chemical reactions accurately such as density functional theory (DFT) [10], Density function based tight binding(DFTB) [11] and so on. Since the DFTB method would be faster a couple of orders of magnitude than DFT, it is more suitable for lager systems.

The non-equilibrium molecular dynamics (NEMD) can reveal a lot of microscopic details of 
shock wave structure. ${ }^{[12]}$ However, the scale of simulation must meet the requirements ${ }^{\text {of }}$ real-scale which also depends on the speed of shock wave. The multiscale shock technique (MSST) ${ }^{[13]}$ is a simulation method based on Navier-Stokes equations for compressive flow. The computational cell could be simulated with fewer atoms and lower computational cost by following a Lagrangian point through the shock wave. ${ }^{[14]}$ Currently, the molecular dynamics method based on MSST have been applied in $\mathrm{HMX}^{[15]}$, TATB ${ }^{[16]}$, and graphite ${ }^{[17]}$ and so on.

In this work we report DFTB-MD simulation of the shock Hugoniot of water, using MSST module in LAMMPS ${ }^{[18]}$ in conjunction with DFTB+ ${ }^{[19]}$ software package. We perform simulations of the following shock velocities $(\mathrm{km} / \mathrm{s}): 5,6.5,7.5,8,8.7,9,10$ and 11 , which is near the experimentally measured steady detonation shock speed. An initial configuration of $64 \mathrm{H}_{2} \mathrm{O}$ molecules with lattice vectors of $a=19.72 \AA, b=9.86 \AA, c=9.86 \AA, \alpha=90^{\circ}, \beta=90^{\circ}$ and $\delta=90^{\circ}$. The direction of uniaxial compression should be along the $a$ lattice vector. For all simulations, a fictitious box mass was set to $3.5 \times 10^{6}$ a.u. and a wave function convergence criteria was set to $10^{-6}$ a.u.

\section{Results and Discussion}

At 7.5 and $8 \mathrm{~km} / \mathrm{s}$, the chemical equilibrium was observed 4 ps after shock compression. When the speed increases up to $8.7 \mathrm{~km} / \mathrm{s}$, the time of chemical equilibrium dropped to approximately 3 ps (Fig.1). At the conditions of this speed, atomic oxygen was observed to form approximately after 1 ps. At a shock velocity of $11 \mathrm{~km} / \mathrm{s}$, the chemical equilibrium, such as temperature and density, of system could be achieved in 2 ps after shock compression. As shown in Figure 4, $\mathrm{H}_{2} \mathrm{O}$ molecules, $\mathrm{H}^{+}, \mathrm{OH}^{-}$and $\mathrm{O}^{2-}$ ions were observed after shock compression. Very few $\mathrm{H}_{3} \mathrm{O}^{+}$species were observed at the initial stage of shock compression. However, as the system went to chemical equilibrium the concentration of ions would decline.

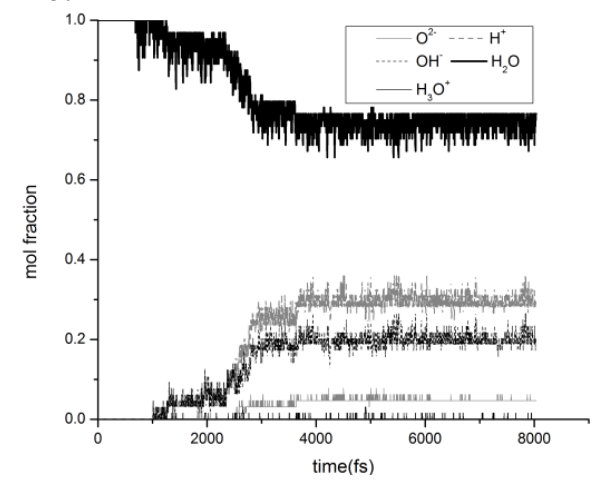

Fig.1 Time dependence of mole fraction of chemical species at $8.7 \mathrm{~km} / \mathrm{s}$

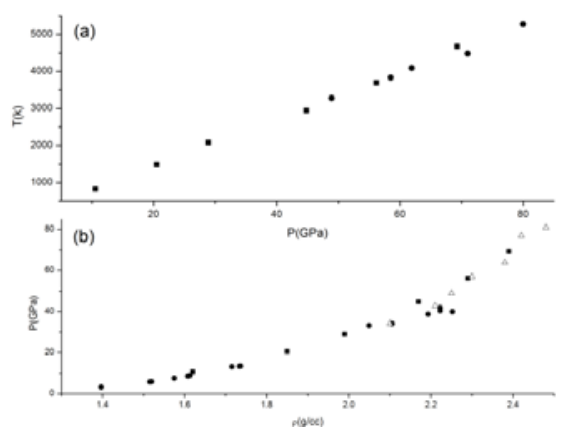

Fig.2 (a) Plot of the temperature vs pressure Hugoniont results. (b) Plot of pressure vs density Hugoniot results.

Our results for the shock Hugoniot of water are shown in Figure 2. As shown in Figure (a), our simulation results are shown with squares while the circles are experimental results from Lyzenga et al. ${ }^{[20]}$ as shown in Figure (b), the squares correspond to our DFTB-MD results, the triangles are experimental results from Walsh et al. ${ }^{[21]}$ and the circles are from Mitchell. ${ }^{[22]}$ Our results provide validation of DFTB-MSST with the generalized gradient approximation equation of state over a wide range of pressures (approximately 10-69 GPa). 


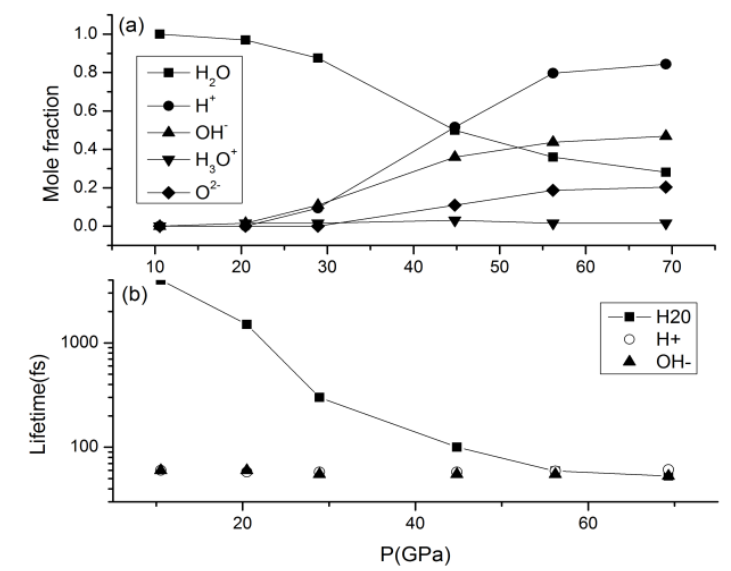

Fig.3 (a) Species concentrations as a function of pressure. (b)Calculated molecular lifetime of ions.

The results for the species concentrations and lifetimes are shown in Figure 3. At $20.5 \mathrm{GPa}$ $(6.5 \mathrm{~km} / \mathrm{s})$ we largely observe reversible unimolecular dissociation in spite of the existence of a small amount of $\mathrm{H}_{3} \mathrm{O}^{+}$after shock compression. As we compress to $44.8 \mathrm{GPa}(9 \mathrm{~km} / \mathrm{s})$, the lifetimes and concentrations of $\mathrm{H}_{2} \mathrm{O}, \mathrm{H}^{+}$, and $\mathrm{OH}^{-}$are all roughly equivalent. Increasing the bond lifetime criteria above $50 \mathrm{fs}$ at this pressure removed all indications of $\mathrm{H}_{3} \mathrm{O}^{+}$and decreased the mole fraction of $\mathrm{H}_{2} \mathrm{O}$ from 0.5 to less than 0.25 . As the simulations were further compressed to $69.3 \mathrm{GPa}$ (11 $\mathrm{km} / \mathrm{s}$ ), equilibration to $\mathrm{H}^{+}, \mathrm{OH}^{-}$and $\mathrm{O}^{2-}$ are observed at sub picosecond, where the lifetimes of all species are vanishingly small. Analysis of the chemical reactivity for all simulations revealed that a unimolecular dissociation mechanism of $\mathrm{H}_{2} \mathrm{O} \leftrightarrow \mathrm{H}^{+}+\mathrm{OH}^{-}$dominates with our bond distance cutoff.

\section{Summary}

All simulations of water under shock loading were performed by our own code, which is utilized the SCC-DFTB from DFTB+ code in conjunction with the MSST from LAMMPS program. Our DFTB-MD simulations show agreement with experimental results for shock Hugoniot pressures, temperatures, and densities. Our results provide validation of DFTB with the generalized gradient approximation for the equation of water at extreme conditions. Determination of the chemical reactions in our simulations indicates that a unimolecular dissociation mechanism of $\mathrm{H}_{2} \mathrm{O} \leftrightarrow \mathrm{H}^{+}+\mathrm{OH}^{-}$dominates along the shock Hugoniot.

\section{References}

[1] KWAK H , SHIN H Y, BAE S Y.Characteristics andkinetics of degradation of polystyrene in supercritical water[ J] .J Applied Polymer Science , 2006, 101(1):695 -700.

[2] Boehler R., Temperatures in the Earth's core from melting-point measurements of iron at high static pressures. Nature, 363,534.

[3] Celliers P M, Collins G W, Hicks D G, et al. Electronic conduction in shock-compressed water[J]. Physics of Plasmas (1994-present), 2004, 11(8): L41-L44.

[4] Lyzenga G A, Ahrens T J, Nellis W J, et al. The temperature of shock-compressed water [J]. The Journal of Chemical Physics, 1982, 76(12): 6282-6286.

[5] Holmes N C, Nellis W J, Graham W B, et al. Spontaneous Raman scattering from shocked water [J]. Physical review letters, 1985, 55(22): 2433.

[6] Schwegler E, Galli G, Gygi F, et al. Dissociation of water under pressure [J]. Physical Review Letters, 2001, 87(26): 265501.

[7] Kadau K, Germann T C, Lomdahl P S, et al. Microscopic view of structural phase transitions induced by shock waves [J]. Science, 2002, 296(5573): 1681-1684.

[8] Strachan A, van Duin A C T, Chakraborty D, et al. Shock waves in high-energy materials: The 
initial chemical events in nitramine RDX [J]. Physical Review Letters, 2003, 91(9): 098301.

[9] Kress J D, Bickham S R, Collins L A, et al. Tight-binding molecular dynamics of shock waves in methane[J]. Physical review letters, 1999, 83(19): 3896.

[10]Gygi F, Galli G. Electronic excitations and the compressibility of deuterium [J]. Physical Review B, 2002, 65(22): 220102.

[11]Fihey A, Hettich C, Touzeau J, et al. SCC - DFTB parameters for simulating hybrid gold thiolates compounds [J]. Journal of computational chemistry, 2015, 36(27): 2075-2087.

[12]HeimAJ, JensenNG, KoberEM, GermannTC 2008Phys. Rev. E78046710

[13]Reed E J, Fried L E, Manaa M R, et al. A multi-scale approach to molecular dynamics simulations of shock waves [J]. Chemistry at Extreme Conditions, 2005: 297-326.

[14]Reed E J, Maiti A, Fried L E. Anomalous sound propagation and slow kinetics in dynamically compressed amorphous carbon [J]. Physical Review E, 2010, 81(1): 016607.

[15]Wen Y, Xue X, Zhou X, et al. Twin Induced Sensitivity Enhancement of HMX versus Shock: A Molecular Reactive Force Field Simulation [J]. The Journal of Physical Chemistry C, 2013, 117(46): 24368-24374.

[16]Manaa M R, Reed E J, Fried L E, et al. Nitrogen-rich heterocycles as reactivity retardants in shocked insensitive explosives [J]. Journal of the American Chemical Society, 2009, 131(15): 5483-5487.

[17]Mundy C J, Curioni A, Goldman N, et al. Ultrafast transformation of graphite to diamond: An ab initio study of graphite under shock compression[J]. The Journal of chemical physics, 2008, 128(18): 184701.

[18]Frauenheim T. DFTB+, Density Functional based Tight Binding; Release: 1.0[J]. See http://www. Dftb-plus. Info. There is no corresponding record for this reference, 2008.

[19]The CP2K Developers Group, http://cp2k.berlios.de/, 2007

[20]Lyzenga G A, Ahrens T J, Nellis W J, et al. The temperature of shock - compressed water[J]. The Journal of Chemical Physics, 1982, 76(12): 6282-6286.

[21]Walsh J M, Rice M H. Dynamic compression of liquids from measurements on strong shock waves [J]. The Journal of Chemical Physics, 1957, 26(4): 815-823.

[22] Mitchell A C, Nellis W J. Equation of state and electrical conductivity of water and ammonia shocked to the $100 \mathrm{GPa}$ (1 Mbar) pressure range[J]. The Journal of Chemical Physics, 1982, 76(12): 6273-6281. 